\title{
Fatores Ambientais sobre Escores de Avaliação Visual à Desmama em Bezerros Angus Criados no Rio Grande do Sul ${ }^{1}$
}

\author{
Fernando Flores Cardoso², Ricardo Alberto Cardellino ${ }^{3}$, Leonardo Talavera Campos ${ }^{4}$
}

RESUMO - Os objetivos deste estudo foram determinar a importância dos efeitos de idade da vaca (IV), sexo (S) e idade do bezerro (ID), mês de nascimento (M) e a interação entre idade da vaca e sexo $(\mathrm{IV} * \mathrm{~S})$ sobre escores de avaliação visual (EV) para conformação (CD), precocidade (GD), musculatura (MD) e tamanho (TD) à desmama, de bovinos Angus, e estimar fatores de correção para esses efeitos. Foram utilizados 11.863 registros coletados pelo Programa de Melhoramento de Bovinos de Carne - PROMEBO, durante os anos de 1989 a 1997. Os dados foram analisados por meio do PROC MIXED do SAS. O modelo incluiu os efeitos de grupo de contemporâneos, M, IV, S, ID (linear e quadrático), IV*S e o efeito aleatório do touro. A partir das médias ajustadas de CD, GD, MD e TD, foram estimadas equações de regressão para M e IV*S. Os EV apresentaram resposta aos efeitos de IV, S e ID similar à do ganho de peso na fase de desmama. A idade do bezerro foi o fator responsável pelas maiores variações não-genéticas nos EV. Fatores de correção para ajuste de IV, ID e data juliana do nascimento foram propostos.

Palavras-chave: conformação, idade da vaca, idade do bezerro, musculatura, precocidade, tamanho

\section{Environmental Factors on Visual Evaluation Scores at Weaning of Angus Calves Raised in the State of Rio Grande do Sul - Brazil}

\begin{abstract}
The objectives of this study were to determine the importance of the effects of age of dam (AoD), sex (S) and age of calf (AC), month of birth (M) and the interaction between age of dam and sex (AoD*S) on visual scores (VS) for weaning conformation (WC), precocity (WP), muscling (WM) and size (WS) of Angus cattle, and to estimate correction factors for these effects. Data from 11,863 calves, controlled by the Beef Cattle Improvement Program (PROMEBO), between 1989 and 1997, were used. The data were analyzed using PROC MIXED of SAS. The model included the effects of contemporary group, M, AoD, S, AC (linear and quadratic), AoD*S and the random effect of sire. From the least square means of WC, WP, WM and WS, regression equations for M and AoD*S were estimated. The VS showed response to the effects of AoD, S and AC similar to the weight gain at the weaning phase. The age of calf was the factor responsible for the largest non-genetic variation in VS. Correction factors for AoD, AC and Julian birth date were proposed.
\end{abstract}

Key Words: age of calf, age of dam, conformation, muscling, precocity, size

\section{Introdução}

A pecuária de corte está sob forte pressão para melhorar a qualidade do produto, sem aumentar os custos, em função da concorrência com outras fontes de proteína animal. Nesse sentido, muitos programas de melhoramento adotaram o uso de escores de avaliação visual para estimar a composição da carcaça dos animais e a rapidez com que esses chegarão ao abate.

LONG (1973), ao propor o Sistema de Avaliação Ankony, afirmou que um programa de melhoramento baseado unicamente no ganho de peso não seria adequado, uma vez que a composição do peso (proporção de carne e gordura) não pode ser ignorada, caso deseje-se satisfazer as demandas do mercado. Escores para conformação, musculatura e precoci- dade de terminação são importantes ferramentas disponíveis e economicamente viáveis para a seleção de animais que produzem mais carne ou carcaça mais desejável, em menor tempo (FRIES, 1996).

Os efeitos de ambiente que têm sido considerados importantes na fase de desmama são idade da vaca, sexo e idade do bezerro, além do grupo de contemporâneos, que engloba os efeitos de rebanho, ano, estação e manejo. Muitos estudos foram conduzidos no Brasil (FRIES, 1984; CAMPOS et al., 1989; ELER et al., 1989; MASCIOLI et al., 1996;) e no exterior (CUNDIFF et al., 1966a; ANDERSON e WILLHAM, 1978; LEIGHTON et al., 1982) para determinar a importância e estimar fatores de ajustes para esses efeitos, sobre pesos e ganhos de peso. Entretanto, existe número reduzido de trabalhos so-

\footnotetext{
1 Parte da Dissertação apresentada pelo primeiro autor à FAEM/UFPEL, para obtenção do título de Mestre em Ciências. Financiada pelas CAPES

2 Aluno de Doutorado. Department of Animal Science. Michigan State University. Bolsista da CAPES. E.mail: cardosof@msu.edu

3 Prof. do Departamento de Zootecnia FAEM/UFPEL. CEP 96010-900 Pelotas/RS. Bolsista do CNPq. E.mail: rcard@ufpel.tche.br

4 Associação Nacional de Criadores "Herd Book Collares". E.mail: Itcampos@terra.com.br
} 
bre a importância desses efeitos nas avaliações realizadas por meio de escores visuais.

CARDELLINO e CARDELLINO (1984) analisaram o escore de conformação à desmama em bovinos Hereford e encontraram efeito significativo da idade da vaca e do sexo do bezerro sobre esse caráter. Outro estudo com dados de animais no RS demonstrou efeito significativo da idade da vaca e sexo e ainda destacou a importância da estação de nascimento sobre o escore de conformação à desmama (PONS et al., 1989). Esses estudos não consideraram o efeito da idade do bezerro, no entanto, CARDOSO et al. (1997) encontraram variação importante em conformação, musculatura e tamanho, em função da idade do bezerro à desmama.

A determinação da importância de efeitos ambientais sobre escores de avaliação visual na fase de desmama e a estimativa de fatores de ajuste para esses efeitos são de grande importância para aumentar a precisão da seleção nos programas de melhoramento genético da raça Angus conduzidos no sul do Brasil. Os objetivos deste estudo foram determinar os efeitos de idade da vaca, sexo e idade do bezerro, mês de nascimento e a interação entre idade da vaca e sexo sobre escores de avaliação visual para conformação, precocidade, musculatura e tamanho à desmama e estimar fatores de correção para ajustar registros individuais para esses efeitos.

\section{Material e Métodos}

O trabalho foi desenvolvido utilizando-se dados de campo coletados rotineiramente pelo Programa de Melhoramento de Bovinos de Carne - PROMEBO da Associação Nacional de Criadores "Herd Book Collares" - ANC, nos rebanhos da raça Angus que participam do programa, durante os anos de 1989 a 1997.

Os escores de avaliação visual (EV) consistem em: conformação (CD), que estima a quantidade de carne na carcaça do animal, quando este for abatido, pelo comprimento e profundidade do corpo, desenvolvimento muscular e harmonia geral do indivíduo; precocidade de terminação (GD), que representa a capacidade de armazenar gordura, indicando rapidez para atingir o acabamento; musculatura (MD), que avalia o grau de desenvolvimento muscular apresentado pelo animal; e tamanho (TD), que representa o comprimento e altura do indivíduo. As avaliações foram feitas em uma escala de 1 a 5 , em que cinco representa o grau máximo de expressão do caráter, relativa ao grupo de contemporâneos. Foram consi- derados para a análise somente registros com avaliações completas para os quatro escores, totalizando 11.863 produtos.

Os grupos de contemporâneos foram formados incluindo ano, criador, rebanho, grupo de manejo ou código alimentar e data da pesagem dos animais. Animais com mais de 120 dias de diferença em idade foram considerados em grupo de contemporâneos a parte. Grupos de contemporâneos com menos de dez animais e touros com menos de cinco filhos foram previamente excluídos das análises.

O efeito do mês de nascimento foi incluído no modelo para considerar variações ambientais que ocorrem dentro de época de nascimento. Os meses com menor número de nascimentos (dezembro-janeiro e maio-junho) foram agrupados e os meses com maior número de nascimentos (abril, agosto, setembro, outubro e novembro), divididos em quinzenas, para permitir melhor balanço no número de informações nas subclasses e, conseqüentemente, melhor ajuste dos dados.

Não se considerou, na análise, o efeito de época de nascimento, pelo fato de não ter sido detectado efeito significativo dessa variável em uma análise prévia dos dados (CARDOSO, 1999).

Os dados foram editados e analisados usando-se o programa SAS - Statistical Analysis System (SAS, 1996), por meio do seguinte modelo:

$$
\begin{gathered}
\mathrm{y}_{\mathrm{ijkmno}}=\mu+\mathrm{GC}_{\mathrm{i}}+\mathrm{M}_{\mathrm{j}}+\mathrm{IV}_{\mathrm{k}}+\mathrm{S}_{\mathrm{m}}+\mathrm{IV}_{\mathrm{k}} * \mathrm{~S}_{\mathrm{m}}+ \\
\mathrm{ID}+\mathrm{ID}^{2}+\mathrm{T}_{\mathrm{n}}+\mathrm{e}_{\mathrm{ijkmno}}
\end{gathered}
$$

em que $\mathrm{y}_{\mathrm{ijkmno}}$ é o valor observado (CD, GD, MD, TD); $\mu$, a média geral; $\mathrm{GC}_{\mathrm{i}}$, o efeito do grupo de contemporâneos (1-170); $\mathrm{M}_{\mathrm{j}}$, o efeito do mês de nascimento (1-15); $\mathrm{IV}_{\mathrm{k}}$, o efeito da idade da vaca (2-12 anos); $\mathrm{S}_{\mathrm{m}}$, o efeito do sexo (1-M e 2-F); $\mathrm{IV}_{\mathrm{k}} * \mathrm{~S}_{\mathrm{m}}$, o efeito da interação entre a idade da vaca e o sexo do bezerro; ID, o coeficiente de regressão linear para idade do animal (100-300 dias); ID ${ }^{2}$, o coeficiente de regressão quadrático para idade do animal; $\mathrm{T}_{\mathrm{n}}$, o efeito aleatório do touro, e $\mathrm{e}_{\mathrm{ijkmno}}$, o erro residual.

A análise de variância foi realizada pelo PROC MIXED (SAS, 1996). Os testes de efeitos fixos do modelo foram realizados pelo teste F tipo III. Após a análise de variância, as médias de quadrados mínimos obtidas para idade da vaca foram utilizadas em análises de regressão, obtendo-se equações quadráticas de predição para os caracteres estudados, em função desse efeito. A interação IV*S foi significativa para CD e GD, optando-se por estimar o efeito IV dentro de $\mathrm{S}$ para esses caracteres.

A partir das médias de quadrados mínimos, calcu- 
ladas para mês de nascimento, foram ajustados polinômios de terceiro grau para predizer a variação de $\mathrm{CD}, \mathrm{GD}, \mathrm{MD}$ e TD, em função das variações ambientais no decorrer do ano e que não são explicadas somente pela estação de nascimento. A data juliana média de nascimento dentro de cada classe de mês de nascimento foi utilizada para determinar as equações.

Os valores preditos pelas equações obtidas para mês de nascimento, idade da vaca e idade à desmama foram utilizados no cálculo de fatores de correção multiplicativos para esses efeitos. Devido à variação nas médias dos rebanhos para os caracteres estudados, fatores multiplicativos são mais adequados que os aditivos para correção dos dados (CUNDIFF et al. 1966b). Os fatores de correção foram calculados dividindo-se a média dos valores preditos para o caráter pelo valor específico predito para cada ponto, no caso de mês de nascimento e idade à desmama. Para o caso de idade da vaca, em vez da média dos valores, tomou-se, como base para o cálculo dos fatores, a idade da vaca de oito anos.

\section{Resultados e Discussão}

As médias e os desvios-padrão observados para $\mathrm{CD}, \mathrm{GD}, \mathrm{MD}$ e TD foram, respectivamente, $2,92 \pm 1,10 ; 3,09 \pm 1,08 ; 2,97 \pm 1,06$ e $3,00 \pm 1,06$ unidades de escore. Esses valores estão dentro do esperado, uma vez que os escores são atribuídos em relação ao grupo de contemporâneos e o animal considerado médio recebe escore três.

Todos os efeitos fixos incluídos no modelo foram significativos $(\mathrm{P}<0,05)$, com exceção da interação idade da vaca com sexo do bezerro para MD e TD $(\mathrm{P}>0,40)$. Estes resultados indicam que todos os efeitos normalmente usados para ajustar pesos e ganhos de peso devem também ser considerados para EV, visando aumentar a precisão das estimativas para esses caracteres. O grupo de contemporâneos teve efeito signicativo $(\mathrm{P}<0,0001)$ sobre $\mathrm{EV}$, mesmo com escala de avaliação relativa. Isso se deve, possivelmente, às diferenças de habilidade entre técnicos para atribuir escores com base na média dos animais contemporâneos. Outros autores avaliando escores de conformação em bovinos Hereford, no RS, encontraram efeitos significativos de efeitos ambientais à desmama (CARDELLINO e CARDELLINO, 1984; PONS et al., 1989).

De maneira geral, o comportamento dos efeitos ambientais estudados sobre EV à desmama foi semelhante ao observado no mesmo conjunto de dados, para ganho do nascimento à desmama (CARDOSO, 1999). Também entre os EV, os efeitos de mês de nascimento, idade da vaca, sexo e idade do bezerro tiverem influência similar.

A variação dos EV no decorrer do ano (Figura 1) ocorreu no mesmo sentido da observada por CARDOSO (1999) para ganho do nascimento à desmama. Os piores EV foram observados no mês de fevereiro, concordando com o observado para ganho de peso, entretanto, a magnitude destes valores deve ser vista com cautela dado o baixo número de observações existentes nessa classe. Os melhores EV ocorreram em julho e agosto, indicando que os animais nascidos no início da primavera são favorecidos quanto aos EV. Estes resultados concordam com o trabalho de PONS et al. (1989), os quais observaram que bezerros nascidos entre julho e setembro foram melhor pontuados para conformação que os nascidos nas demais épocas do ano. Para os meses correspondentes ao outono, ocorre o inverso, pois os animais nascidos no início dessa estação apresentam os piores EV.

As equações de predição de CD, GD, MD e TD, em relação à data juliana de nascimento, calculadas a partir da média da data dentro de cada classe de mês de nascimento, são apresentadas na Tabela 1. A partir destas equações, foram estimados fatores de correção para mês de nascimento. Esses fatores estão listados na Tabela 2 e correspondem à média da data dentro de cada classe, entretanto, a partir das equações, podem ser preditos fatores de correção para todas as datas dentro do período. Apesar de

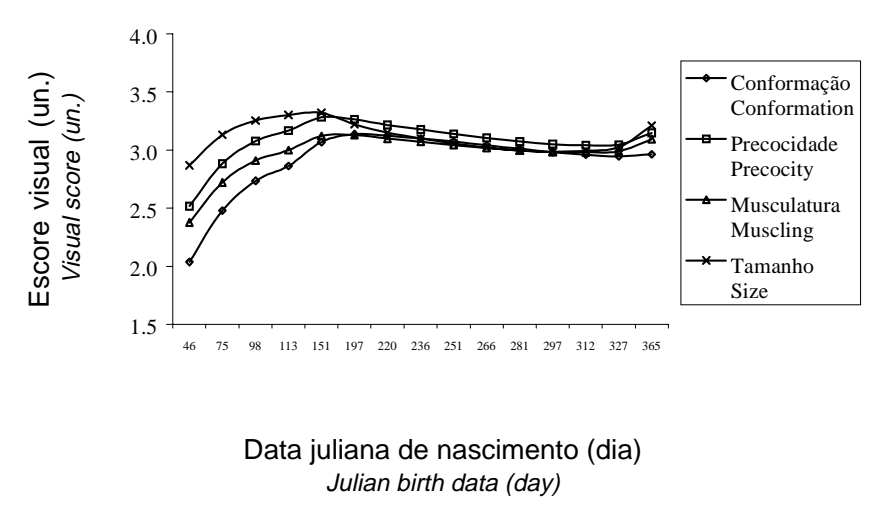

Figura 1 - Curvas estimadas para conformação (CD), precocidade (GD), musculatura (MD) e tamanho (TD) à desmama, de acordo com a data de nascimento juliana.

Figura 1 - Estimated curves for conformation (WC), precocity (WP), muscling (WM) and size (WS) at weaning, according to julian birth date. 
Tabela 1 - Coeficientes das equações de regressão de conformação (CD), precocidade (GD), musculatura (MD) e tamanho (TD) à desmama, em função da data juliana de nascimento, idade da vaca dentro de sexo e idade à desmama Table 1 - Regression equation coefficients of conformation (WC), precocity (WP), muscling (WM) and size (WS) at weaning by julian birth date, age of dam within sex and age at weaning

\begin{tabular}{|c|c|c|c|c|}
\hline \multirow[b]{2}{*}{$\begin{array}{l}\text { Efeito/Caráter } \\
\text { Effect/Trait }\end{array}$} & \multicolumn{4}{|c|}{$\begin{array}{l}\text { Parâmetro } \\
\text { Parameter }\end{array}$} \\
\hline & $\begin{array}{l}\text { Intercepto } \\
\text { Intercept }\end{array}$ & $\begin{array}{l}\text { Linear } \\
\text { Linear }\end{array}$ & $\begin{array}{l}\text { Quadrático } \\
\text { Quadratic }\end{array}$ & $\begin{array}{l}\text { Cúbico } \\
\text { Cubic }\end{array}$ \\
\hline \multicolumn{5}{|l|}{$\begin{array}{l}\text { Data juliana do nascimento } \\
\text { Julian birth date }\end{array}$} \\
\hline $\begin{array}{l}\mathrm{CD}(W C) \\
\mathrm{GD}(W P) \\
\mathrm{MD}(W M) \\
\mathrm{TD}(W S)\end{array}$ & $\begin{array}{l}1,017698038 \\
1,618456847 \\
1,545391186 \\
2,148529459\end{array}$ & $\begin{array}{l}0,026841581 \\
0,024393077 \\
0,022423561 \\
0,020290071\end{array}$ & $\begin{array}{l}-0,000108189 \\
-0,000111920 \\
-0,000100176 \\
-0,000107819\end{array}$ & $\begin{array}{l}0,000000135 \\
0,000000155 \\
0,000000138 \\
0,000000165\end{array}$ \\
\hline \multicolumn{5}{|l|}{$\begin{array}{l}\text { Idade da vaca (anos) } \\
\text { Age of dam (years) }\end{array}$} \\
\hline $\begin{array}{l}\text { CD-Machos (WC-Males) } \\
\text { CD-Fêmeas (WC-Females) } \\
\text { GD-Machos (WP-Males }) \\
\text { GD-Fêmeas (WP-Females) } \\
\text { MD (WM) } \\
\text { TD }(W S)\end{array}$ & $\begin{array}{l}1,861748493 \\
1,963464354 \\
1,948239703 \\
2,308996987 \\
2.081912946 \\
2.135244832\end{array}$ & $\begin{array}{l}0,319419093 \\
0,290376824 \\
0,337083469 \\
0,254081968 \\
0.267846887 \\
0.313120690\end{array}$ & $\begin{array}{l}-0,019745062 \\
-0,018992248 \\
-0,021497281 \\
-0,016248755 \\
-0.016739068 \\
-0.020675001\end{array}$ & \\
\hline \multicolumn{5}{|l|}{$\begin{array}{l}\text { Idade à desmama } \\
\text { Age at weaning }\end{array}$} \\
\hline $\begin{array}{l}\mathrm{CD}(W C) \\
\mathrm{GD}(W P) \\
\mathrm{MD}(W M) \\
\mathrm{TD}(W S) \\
\end{array}$ & $\begin{array}{c}-0,21559248 \\
0,54531520 \\
0,44950139 \\
-1,17097081 \\
\end{array}$ & $\begin{array}{l}0,02081629 \\
0,01761760 \\
0,01697668 \\
0,03102531 \\
\end{array}$ & $\begin{array}{l}-0,00002354 \\
-0,00002242 \\
-0,00002005 \\
-0,00004653 \\
\end{array}$ & \\
\hline
\end{tabular}

serem apresentados valores para o mês de fevereiro neste estudo, dada a baixa acurácia da estimativa da magnitude do efeito deste mês, parece mais recomendável utilizar os mesmos fatores de $1^{\circ}$ de março para corrigir EV de animais nascidos em fevereiro, até que maior volume de informações esteja disponível para obtenção de estimativas mais confiáveis. Considerando os meses de março a dezembro, a maior variação em EV, em função do efeito de mês de nascimento, foi observada em CD $(24,7 \%)$. O caráter visual que menos variou em função do mês de nascimento foi TD (10,5\%).

O efeito do sexo do bezerro foi significativo para todos os $\mathrm{EV}(\mathrm{P}<0,01)$. Isso indica que, na prática, os técnicos não conseguem compensar o efeito de sexo, mesmo avaliando machos e fêmeas separadamente e de forma relativa ao seu grupo de contemporâneos. Outros autores que trabalharam com escores de conformação de animais avaliados pelo PROMEBO também encontraram diferenças significativas entre machos e fêmeas (CARDELLINO e CARDELLINO, 1984; PONS et al., 1989).

As médias ajustadas e os erros-padrão para $\mathrm{CD}$, MD e TD foram, respectivamente, 2,93 $\pm 0,06$;
$3,05 \pm 0,06$ e $3,17 \pm 0,06$ unidades de escore para machos e $2,88 \pm 0,06 ; 2,88 \pm 0,06$ e $3,05 \pm 0,06$ unidades de escore para fêmeas. As diferenças foram de 1,$7 ; 5,5$; e 3,9\% em favor dos machos, respectivamente, para CD, MD e TD. PONS et al. (1989) também encontraram superioridade dos machos em relação às fêmeas de 0,61 pontos ou $5,1 \%$ para escore de conformação. Quanto a GD, as fêmeas apresentaram média ajustada de 3,13 $\pm 0,06$ unidades, sendo essa $3 \%$ superior à dos machos, de 3,04 $\pm 0,06$ unidades. Essa aparente discrepância pode ser explicada pelo menor potencial de crescimento das fêmeas em comparação aos machos. As fêmeas utilizam parcela menor dos nutrientes consumidos para o desenvolvimento corporal e muscular, armazenando mais gordura e apresentando melhor estado corporal à desmama, em relação aos machos. O estado corporal é componente do grau de acabamento avaliado em GD; assim, as fêmeas recebem melhores notas que os machos nesse caráter, comprovando que os técnicos não conseguem compensar totalmente o efeito de sexo em EV.

Apesar de a escala de EV ser relativa ao grupo de animais avaliados dentro de sexo, este efeito 
322 Rev. bras. zootec.

Tabela 2 - Fatores de correção multiplicativos para conformação (CD), precocidade (GD), musculatura (MD) e tamanho (TD) à desmama, em função do mês de nascimento

Table 2 - Multiplicative correction factors for conformation (WC), precocity (WP), muscling (WM) and size (WS) at weaning by month of birth

\begin{tabular}{|c|c|c|c|c|}
\hline \multirow{3}{*}{$\begin{array}{l}\text { Mês de nascimento } \\
\text { Month of birth }\end{array}$} & \multicolumn{4}{|c|}{$\begin{array}{c}\text { Fatores de correção multiplicativos } \\
\text { Multiplicative correction factors }\end{array}$} \\
\hline & CD (un.) & GD (un.) & MD (un.) & TD (un.) \\
\hline & WC (un.) & $W P($ un.) & $W M($ un.) & WS (un.) \\
\hline${\overline{F e v e r e i r o ~}(46)^{1}}^{1}$ & 1,4251 & 1,2230 & 1,2487 & 1,0838 \\
\hline February $(46)^{1}$ & & & & \\
\hline $\begin{array}{l}\text { Março (75) } \\
\text { March (75) }\end{array}$ & 1,1707 & 1,0682 & 1,0911 & 0,9926 \\
\hline $\begin{array}{l}\text { 1-15/Abril (98) } \\
\text { April, 1-15 (98) }\end{array}$ & 1,0607 & 1,0002 & 1,0203 & 0,9551 \\
\hline $\begin{array}{l}\text { 16-30/Abril(113) } \\
\text { April, 16-30 (113) }\end{array}$ & 1,0134 & 0,9720 & 0,9902 & 0,9418 \\
\hline $\begin{array}{l}\text { Maio/Junho (151) } \\
\text { May/June (151) }\end{array}$ & 0,9458 & 0,9382 & 0,9512 & 0,9363 \\
\hline $\begin{array}{l}\text { Julho (197) } \\
\text { July (197) }\end{array}$ & 0,9246 & 0,9434 & 0,9488 & 0,9651 \\
\hline $\begin{array}{l}\text { 1-15/Agosto (220) } \\
\text { August, 1-15 (220) }\end{array}$ & 0,9291 & 0,9572 & 0,9582 & 0,9872 \\
\hline $\begin{array}{l}\text { 16-31/Agosto (236) } \\
\text { August, 16-31(236) }\end{array}$ & 0,9359 & 0,9690 & 0,9668 & 1,0031 \\
\hline $\begin{array}{l}\text { 1-15/Setembro }(251) \\
\text { September, } 1-15(251)\end{array}$ & 0,9443 & 0,9807 & 0,9754 & 1,0172 \\
\hline $\begin{array}{l}\text { 16-30/Setembro (266) } \\
\text { September, } 16-30(266)\end{array}$ & 0,9537 & 0,9921 & 0,9836 & 1,0291 \\
\hline $\begin{array}{l}\text { 1-15/Outubro }(281) \\
\text { October, } 1-15(281)\end{array}$ & 0,9633 & 1,0019 & 0,9905 & 1,0376 \\
\hline $\begin{array}{l}\text { 16-31/Outubro (297) } \\
\text { October, 16-31(297) }\end{array}$ & 0,9729 & 1,0095 & 0,9952 & 1,0414 \\
\hline $\begin{array}{l}\text { 1-15/Novembro (312) } \\
\text { November, } 1-15 \text { (312) }\end{array}$ & 0,9803 & 1,0127 & 0,9962 & 1,0386 \\
\hline $\begin{array}{l}\text { 16-30/Novembro }(327) \\
\text { November, } 16-30(327)\end{array}$ & 0,9849 & 1,0110 & 0,9928 & 1,0286 \\
\hline $\begin{array}{l}\text { Dezembro/Janeiro (365) } \\
\text { December/January (365) }\end{array}$ & 0,9785 & 0,9784 & 0,9597 & 0,9679 \\
\hline
\end{tabular}

${ }^{1}$ Data juliana usada para cálculo do fator de correção.

${ }^{1}$ Julian date used for calculation of correction factor.

não é eliminado completamente e deve ser considerado nas avaliações genéticas. Estes resultados contrariam o trabalho de CARDOSO et al. (1997), com dados de animais Santa Gertrudis, avaliados pelo PROMEBO, que concluíram que o efeito de sexo sobre EV não é importante. Como machos e fêmeas recebem pressões de seleção bastante diferentes em bovinos de corte, recomenda-se a inclusão do sexo dentro do grupo de contemporâneos para o controle desse efeito.

A idade da vaca influenciou significativamente todos os EV ( $\mathrm{P}<0,0001)$. O efeito da idade da vaca sobre EV no presente estudo (Figura 2) foi semelhante ao encontrado porCARDELLINOe CARDELLINO(1984) sobre escore de conformação à desmama e demonstra que o efeito da idade da vaca afeta o desenvolvimento corporal dos bezerros, medido por meio de EV, da mesma forma em que afeta o seu crescimento.

Seguindo a mesma metodologia empregada em CARDOSO (1999) para ganho do nascimento à desmama, a partir das médias ajustadas, foram estimadas equações de predição para EV pela idade da vaca, considerando-se esse efeito dentro de sexo no caso de CD e GD. A forma mais acentuada da curva de desempenho de machos em CD e GD, com relação à idade da vaca (Figura 2), demonstra que machos são mais susceptíveis que fêmeas ao desempenho materno inferior das novilhas. Os machos filhos de novilhas foram inferiores às fêmeas em $\mathrm{CD}$, mas aqueles filhos de vacas maduras foram superiores nesse caráter. As fêmeas filhas de vacas de todas as idades foram superiores aos machos em GD, embora as diferenças 


\section{CARDOSO et al.}

nos filhos de vacas maduras tenham sido menores.

Os fatores de correção para EV pela idade da vaca são apresentados na Tabela 3. Para cálculo desses fatores, a idade da vaca de oito anos, em que se encontraram os maiores desempenhos, foi considerada o ponto base. As maiores diferenças de desempenho em EV, em função da idade da vaca, foram encontradas em CD $(23,2 \%)$ e GD $(22,4 \%)$ para machos filhos de novilhas de dois anos, em comparação com vacas adultas de oito anos. A magnitude desses fatores foi superior à encontrada nos estudos de CARDELLINO e CARDELLINO (1984) e PONS et al. (1989), para conformação à desmama. O efeito da idade da vaca sobre EV foi maior que o observado sobre G205 no mesmo conjunto de dados, ressaltando a importância de ajustar os registros de CD, GD, MD e TD para maior precisão das avaliações genéticas desses caracteres na fase de desmama.

A idade à desmama foi, entre os efeitos estudados, a causa individual de maior variação nos EV (Figura 3), o que se deve ao fato de nenhum ajuste prévio ser realizado para esses caracteres. As equações estimadas diretamente na base de dados, pela inclusão desse efeito como covariável no modelo, para predição de CD, GD, MD e $\mathrm{TD}$, em função da idade do bezerro à desmama, são apresentadas na Tabela 1. Bezerros mais jovens apresentam menor grau de desenvolvimento corporal à desmama em comparação aos mais velhos, recebendo piores escores na avaliação morfológica visual.
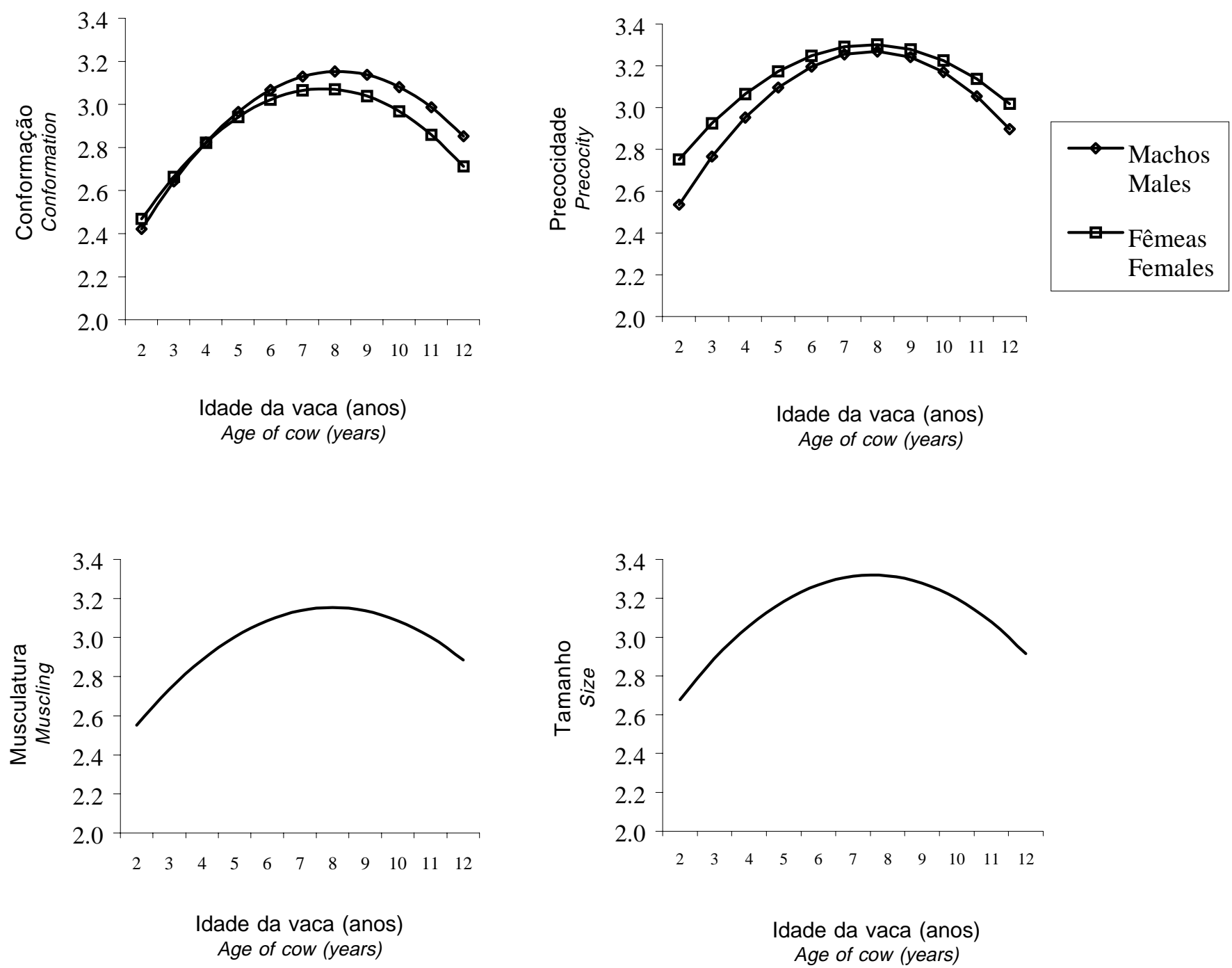

Figura 2 - Curvas estimadas para conformação (CD), precocidade (GD), musculatura (MD) e tamanho (TD) à desmama, de acordo com a idade da vaca.

Figura 2 - Estimated curves for conformation (WC), precocity (WP), muscling (WM) and size (WS) at weaning), according to age of dam. 
324 Rev. bras. zootec.

Tabela 3 - Fatores de correção multiplicativos para conformação (CD), precocidade (GD), musculatura (MD) e tamanho (TD) à desmama pela idade da vaca

Table 3 - Multiplicative correction factors for conformation (WC), precocity (WP), muscling (WM) and size (WS) at weaning by age of dam

Fatores de correção multiplicativos

Multiplicative correction factors

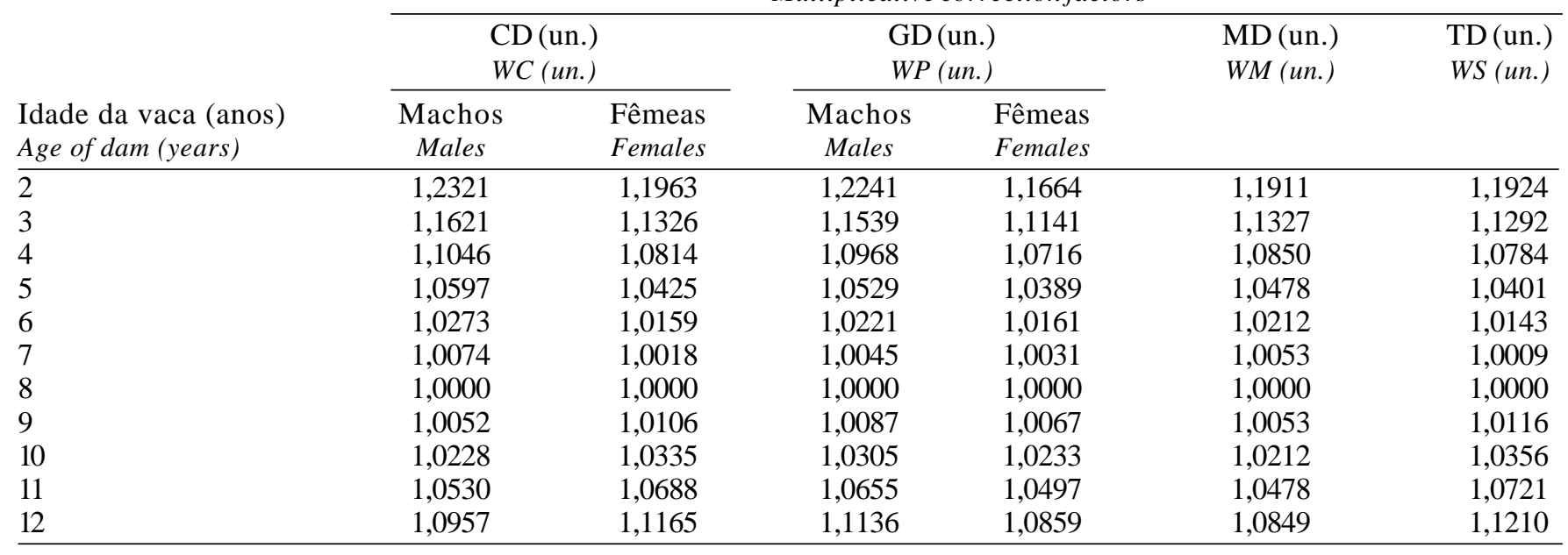

No presente conjunto de dados, as maiores variações foram observadas em CD e TD, de 2,28 e 2,48 unidades de EV, respectivamente, entre 100 e 300 dias de idade do bezerro à desmama. Para GD e MD, essas mesmas diferenças foram levemente menores, sendo 1,73 e 1,79 unidades, respectivamente, porém não diminuem em importância e representam incremento superior a $65 \%$. Estes valores evidenciam a importância de considerar o efeito da idade à desmama, ao selecionar animais pelos EV.

PONS et al. (1989) não consideraram o efeito da idade em suas análises, mas observaram que os bezerros nascidos na primavera receberam escores de conformação acentuadamente maiores que os nascidos no verão e atribuíram estas diferenças à maior idade dos primeiros no momento da desmama. CARDOSO et al.(1997)encontraram efeito significativo da idade à desmama sobre conformação, musculatura e tamanhoà desmama, emanimais daraça Santa Gertrudis criados nas mesmas condições do presente estudo.

Ao contrário do que ocorre com caracteres ponderais, não é lógico prever incremento diário de escores, nem existe um valor de escore ao nascimento para ajustar escores para ganho diário. Assim, fatores de correção multiplicativos são propostos para ajustar CD, GD, MD e TD, em função da idade dos bezerros. Apesar de os fatores serem listados na Tabela 4 em intervalos de dez dias de idade, a partir das equações de predição, é possível obter fatores para correção de todas as idades entre 100 e 300 dias.
O uso desses fatores irá certamente aumentar a precisão nas comparações dos animais em termos de $\mathrm{CD}, \mathrm{GD}, \mathrm{MD}$ e TD à desmama. A correção para idade também é fundamental para a comparação correta de touros, sendo particularmente importante em uma situação que ocorre comumente no RS, quando é usado um período de inseminação artificial seguido de acasalamento com reprodutores em monta natural. Nesse caso, os touros usados em inseminação

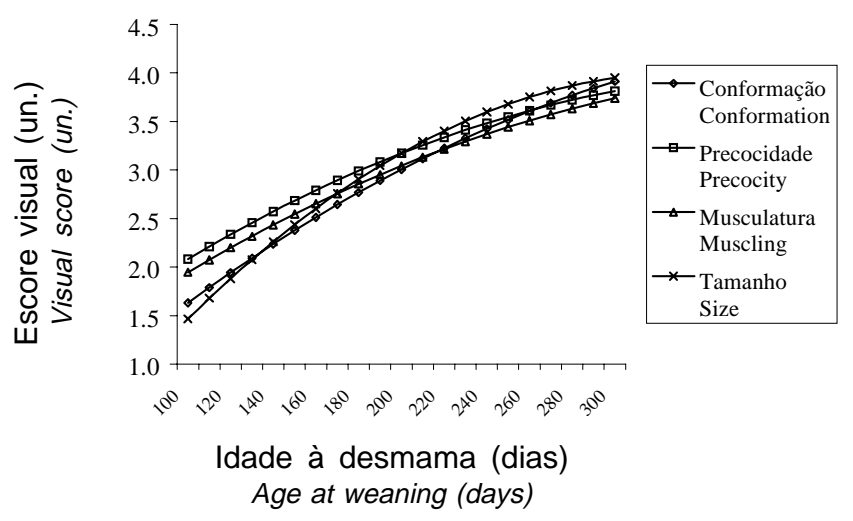

Figura 3 - Curvas estimadas para conformação (CD), precocidade (GD), musculatura (MD) e tamanho (TD) à desmama, de acordo com a idade à desmama.

Figura 3 - Estimated curves for conformation (WC), precocity $(W P)$, muscling (WM) and size (WS) at weaning, according to age at weaning. 
Tabela 4 - Fatores de correção multiplicativos para conformação (CD), precocidade (GD), musculatura (MD) e tamanho (TD) à desmama em função da idade do bezerro à desmama (ID)

Table 4 - Multiplicative correction factors for conformation $(W C)$, precocity (WP), muscling (WM) and size (WS) at weaning by age of calf at weaning (AC)

\begin{tabular}{|c|c|c|c|c|}
\hline $\begin{array}{l}\text { Idade do bezerro } \\
\text { à desmama (dias) }\end{array}$ & $\mathrm{CD}$ (un.) & GD(un.) & MD (un.) & $\mathrm{TD}$ (un.) \\
\hline $\begin{array}{l}\text { Age of calfat } \\
\text { weaning (days) }\end{array}$ & $W C($ un.) & $W P($ un.) & $W M($ un.) & WS (un.) \\
\hline 100 & 1,7906 & 1,4834 & 1,5253 & 2,0476 \\
\hline 110 & 1,6317 & 1,3969 & 1,4315 & 1,7883 \\
\hline 120 & 1,5024 & 1,3224 & 1,3509 & 1,5952 \\
\hline 130 & 1,3952 & 1,2577 & 1,2812 & 1,4462 \\
\hline 140 & 1,3050 & 1,2012 & 1,2203 & 1,3281 \\
\hline 150 & 1,2282 & 1,1514 & 1,1668 & 1,2325 \\
\hline 160 & 1,1621 & 1,1074 & 1,1194 & 1,1539 \\
\hline 170 & 1,1048 & 1,0683 & 1,0774 & 1,0883 \\
\hline 180 & 1,0546 & 1,0334 & 1,0398 & 1,0331 \\
\hline 190 & 1,0104 & 1,0021 & 1,0061 & 0,9863 \\
\hline 200 & 0,9713 & 0,9741 & 0,9758 & 0,9462 \\
\hline 210 & 0,9365 & 0,9489 & 0,9485 & 0,9119 \\
\hline 220 & 0,9054 & 0,9262 & 0,9239 & 0,8824 \\
\hline 230 & 0,8776 & 0,9058 & 0,9016 & 0,8570 \\
\hline 240 & 0,8526 & 0,8873 & 0,8814 & 0,8351 \\
\hline 250 & 0,8301 & 0,8708 & 0,8630 & 0,8165 \\
\hline 260 & 0,8098 & 0,8558 & 0,8464 & 0,8006 \\
\hline 270 & 0,7915 & 0,8425 & 0,8314 & 0,7872 \\
\hline 280 & 0,7750 & 0,8305 & 0,8178 & 0,7762 \\
\hline 290 & 0,7601 & 0,8198 & 0,8055 & 0,7672 \\
\hline 300 & 0,7466 & 0,8104 & 0,7944 & 0,7603 \\
\hline
\end{tabular}

seriam favorecidos em comparação com os reprodutores de monta natural, pela maior idade de seu filhos no momento da desmama.

\section{Conclusões}

Deve-se considerar o efeito do mês ou da data juliana do nascimento, nas avaliações de CD, GD, MD e TD, pois a inclusão do grupo de contemporâneos no modelo estatístico não elimina os efeitos sazonais no decorrer do ano.

Os ajustes de CD e GD pela idade da mãe devem ser feitos dentro de sexo, para assim evitar que bezerros machos filhos de novilhas sejam prejudicados em relação às fêmeas.

A idade do bezerro, como fator responsável pelas maiores variações não genéticas nos EV, deve ser considerada nas avaliações genéticas de CD, GD, MD e TD.

\section{Referências Bibliográficas}

ANDERSON, J.H., WILLHAM, R.L. 1978. Weaning weight correction factors from Angus field data. J. Anim. Sci., 47(1):124-130.
CAMPOS, L.T., SILVA, P.R., FRIES, L.A. 1989. Fatores de correção para efeitos ambientais que afetam o ganho do nascimento à desmama em bovinos da raça Nelore. Coletânea de Pesquisas Inéditas sobre Zebu. Empresa de Pesquisa Agropecuária de Minas Gerais. EPAMIG. Uberaba, MG. p.108-123.

CARDELLINO, M.V., CARDELLINO R.A. 1984. Efeitos ambientais sobre peso, ganho de peso e conformação à desmama em bovinos Hereford no Rio Grande do Sul. R. Soc. Bras. Zootec., 13(4):547-556.

CARDOSO, F.F. Caracterização genética do desempenho do nascimento à desmama de bovinos Angus criados no Rio Grande do Sul. Pelotas,RS:UFPEL, 1999.116p. Dissertação (Mestrado) - Faculdade de Agronomia Eliseu Maciel/Universidade Federal de Pelotas, 1999.

CARDOSO, F.F., CARDELLINO, R.A., CAMPOS, L.T. Efeitos ambientais sobre caracteres produtivos de bovinos da raça Santa Gertrudis. In: CONGRESSO BRASILEIRO DE MEDICINA VETERINÁRIA, 25, 1997, Gramado. Anais... Gramado: SOVERGS, 1997. p.256.

CUNDIFF, L.V., WILlHAM, R.L., PRATT, C.A. 1966 a. Effects of certain factors and their two way interactions on weaning weights in beef cattle. J. anim. Sci., 25(4):972-982.

CUNDIFF, L.V., WILlHAM, R.L., PRATT, C.A. 1966b. Additive versus multiplicative correction factors for weaning weights in beef cattle. J. Anim. Sci., 25(4):983-987.

ELER, J.P., LOBO, R.B., ROSA, A.N. 1989. Influência de fatores genéticos e de meio em pesos de bovinos da raça Nelore criados no estado de São Paulo. R. Soc. Bras. Zootec., 18(2):103-111.

FRIES, L.A. A study of weaning weights in Hereford cattle in the state of Rio Grande do Sul, Brazil. Ames, 1984.317p. Thesis (Ph.D.) - Iowa State University, 1984.

FRIES, L.A. Uso de escores visuais em programas de seleção para a produtividade em gado de corte. In: SEMINÁRIO NACIONAL - REVISÃO DE CRITÉRIO DE JULGAMENTO E SELEÇÃO EM GADO DE CORTE, 1996, Uberaba. Anais... Uberaba:ABCZ, 1996. p.1-6.

LEIGHTON, E.A., WILLHAM, R.L, BERGER P.J. 1982. Factors influencing weaning weight in Hereford cattle and adjustment factors to correct records for these effects. J. Anim. Sci., 54(5):957-963.

LONG, R.A. 1973. El Sistema de Evaluación de Ankony y su aplicación en la mejora del ganado. Ankony Corporation, Grand Juntion, Colorado. 21p.

MASCIOLI, A.S., ALENCAR, M.M., BARBOSA, P.F. et al. 1996. Influência de fatores de meio sobre pesos de animais da raça Canchim. R. Soc. Bras. Zootec., 25(5):853-865.

PONS, S.B., MILAGRES J.C., TEIXEIRA, N.M. 1989. Efeitos de fatores genéticos e de ambiente sobre o crescimento e o escore de conformação em bovinos Hereford no Rio Grande do Sul. I - Peso e escore de conformação à desmama. R. Soc. Bras. Zootec., 18(5):391-401.

SAS INSTITUTE INC. SAS/STAT. User' Guide, release 6.11 Edition. Cary: SAS Institute Inc. 1996.

Recebido em: 23/08/99

Aceito em: 20/11/00 